\title{
Universal suspension via noncommutative motives
}

\author{
Gonçalo Tabuada*
}

\begin{abstract}
In this article we further the study of noncommutative motives, initiated in [5], [6], [28]. Our main result is the construction of a simple model, given in terms of infinite matrices, for the suspension in the triangulated category of noncommutative motives. As a consequence, this simple model holds in all the classical invariants such as Hochschild homology, cyclic homology and its variants (periodic, negative, ... .), algebraic K-theory, topological Hochschild homology, topological cyclic homology, etc.
\end{abstract}

Mathematics Subject Classification (2010). 18D20, 19D35, 19 D55.

Keywords. Noncommutative motives, infinite matrix algebras, algebraic K-theory, cyclic homology, topological Hochschild homology.

\section{Introduction}

Noncommutative motives. A differential graded $(\mathrm{dg})$ category over a commutative base ring $k$ is a category enriched over complexes of $k$-modules (morphisms sets are such complexes) in such a way that composition satisfies the Leibniz rule: $d(f \circ g)=(d f) \circ g+(-1)^{\operatorname{deg}(f)} f \circ(d g)$. Dg categories enhance and solve many of the technical problems inherent to triangulated categories; see Keller's ICM address [16]. In noncommutative algebraic geometry in the sense of Bondal, Drinfeld, Kaledin, Kapranov, Kontsevich, Toën, Van den Bergh, and others [2], [3], [7], [10], [17], [18], [31], they are considered as dg-enhancements of derived categories of (quasi-)coherent sheaves on a hypothetic noncommutative space.

All the classical (functorial) invariants, such as Hochschild homology $\mathrm{HH}$, cyclic homology HC, (non-connective) algebraic K-theory $\mathbb{K}$, topological Hochschild homology THH, and topological cyclic homology TC, extend naturally from $k$-algebras to dg categories. In order to study all these classical invariants simultaneously the author introduced in [28] the notion of localizing invariant. This notion that we now recall makes use of the language of Grothendieck derivators [9], a formalism which allows us to state and prove precise universal properties. Let $L: \mathrm{HO}$ (dgcat) $\rightarrow \mathbb{D}$ be a morphism of derivators from the derivator associated with the derived Morita

\footnotetext{
* The author was partially supported by the Estimulo à Investigação Award 2008 - Calouste Gulbenkian Foundation and by the FCT-Portugal grant PTDC/MAT/098317/2008.
} 
model structure on dg categories (see \$2.2) to a triangulated derivator. We say that $L$ is a localizing invariant if it preserves filtered homotopy colimits as well as the terminal object, and sends exact sequences of dg categories (see §2.3)

$$
\mathcal{A} \rightarrow \mathscr{B} \rightarrow \mathcal{C} \mapsto L(\mathcal{A}) \rightarrow L(\mathscr{B}) \rightarrow L(\mathcal{C}) \rightarrow L(\mathcal{A})[1]
$$

to distinguished triangles in the base category $\mathbb{D}(e)$ of $\mathbb{D}$. Due to the work of Keller [15], [13], Thomason-Trobaugh [30], Schlichting [22], and Blumberg-Mandell [1] (see also [29]) all the mentioned invariants satisfy localization ${ }^{1}$, and so give rise to localizing invariants. In [28], the author constructed the universal localizing invariant

$$
u_{\mathrm{dg}}^{\text {loc }}: \mathrm{HO}(\text { dgcat }) \rightarrow \text { Mot }_{\mathrm{dg}}^{\text {loc }},
$$

i.e., given any triangulated derivator $\mathbb{D}$, we have an induced equivalence of categories

$$
\left(\mathcal{U}_{\mathrm{dg}}^{\text {loc }}\right)^{*}: \underline{\mathrm{Hom}}_{!}\left(\operatorname{Mot}_{\mathrm{dg}}^{\text {loc }}, \mathbb{D}\right) \stackrel{\sim}{\longrightarrow} \underline{\mathrm{Hom}}_{\mathrm{loc}}(\mathrm{HO}(\text { dgcat }), \mathbb{D}),
$$

where the left-hand side denotes the category of homotopy colimit preserving morphisms of derivators, and the right-hand side denotes the category of localizing invariants. Because of this universality property, which is a reminiscence of motives, $\operatorname{Mot}_{\mathrm{dg}}^{\text {loc }}$ is called the localizing motivator, and its base category $\operatorname{Mot}_{\mathrm{dg}}^{\text {loc }}(e)$ the category of noncommutative motives. We invite the reader to consult [5], [6], [28] for several applications of this theory of noncommutative motives.

Universal suspension. The purpose of this article is to construct a simple model for the suspension in the triangulated category of noncommutative motives.

Consider the $k$-algebra $\Gamma$ of $\mathbb{N} \times \mathbb{N}$-matrices $A$ which satisfy the following two conditions: (1) the set $\left\{A_{i, j} \mid i, j \in \mathbb{N}\right\}$ is finite; (2) there exists a natural number $n_{A}$ such that each row and each column has at most $n_{A}$ non-zero entries; see Definition 3.5. Let $\Sigma$ be the quotient of $\Gamma$ by the two-sided ideal consisting of those matrices with finitely many non-zero entries; see Definition 3.1. Alternatively, take the (left) localization of $\Gamma$ with respect to the matrices $\overline{I_{n}}, n \geq 0$, with entries $\left(\overline{I_{n}}\right)_{i, j}=\mathbf{1}$ for $i=j>n$ and 0 otherwise; see Proposition 3.11. The algebra $\Sigma$ goes back to the work of Karoubi and Villamayor [11] on negative K-theory. Recently, it was used by Cortiñas and Thom [4] in the construction of a bivariant algebraic K-theory. Given a dg category $\mathcal{A}$, we denote by $\Sigma(\mathcal{A})$ the tensor product of $\mathcal{A}$ with $\Sigma$; see $\S 2.1$. The main result of this article is the following.

Theorem 1.2. For every dg category $A$ we have a canonical isomorphism

$$
\mathcal{U}_{\mathrm{dg}}^{\mathrm{loc}}(\Sigma(\mathcal{A})) \stackrel{\sim}{\longrightarrow} \mathcal{U}_{\mathrm{dg}}^{\text {loc }}(\mathcal{A})[1] .
$$

The proof of Theorem 1.2 is based on several properties of the category of noncommutative motives (see Section 6), on an exact sequence relating $\mathcal{A}$ and $\Sigma(\mathcal{A})$ (see Section 4), and on the flasqueness of $\Gamma$ (see Section 5). Let us now describe some applications of Theorem 1.2.

\footnotetext{
${ }^{1}$ In the case of algebraic K-theory we consider its non-connective version.
} 
Applications. A realization of the category of noncommutative motives is a triangulated functor $R: \operatorname{Mot}_{\mathrm{dg}}^{\text {loc }}(e) \rightarrow \mathcal{T}$. An important aspect of a realization is the fact that every result which holds on $\operatorname{Mot}_{\mathrm{dg}}^{\text {loc }}(e)$ also holds on $\mathcal{T}$. In particular, given a $\mathrm{dg}$ category $\mathcal{A}$, Theorem 1.2 gives us a canonical isomorphism

$$
\left(R \circ \mathcal{U}_{\mathrm{dg}}^{\mathrm{loc}}\right)(\Sigma(\mathcal{A})) \stackrel{\sim}{\longrightarrow}\left(R \circ \mathcal{U}_{\mathrm{dg}}^{\mathrm{loc}}\right)(\mathcal{A})[1] .
$$

Due to the above equivalence (1.1) every localizing invariant gives rise to a realization. Hence we obtain the canonical isomorphisms

$$
\begin{aligned}
\mathrm{HH}(\Sigma(\mathcal{A})) & \simeq \mathrm{HH}(\mathcal{A})[1], & \mathrm{HH}_{*+1}(\Sigma(\mathcal{A})) & \simeq \mathrm{HH}_{*}(\mathcal{A}), \\
\mathrm{HC}(\Sigma(\mathcal{A})) & \simeq \mathrm{HC}(\mathcal{A})[1], & \mathrm{HC}_{*+1}(\Sigma(\mathcal{A})) & \simeq \mathrm{HC}_{*}(\mathcal{A}), \\
\mathbb{K}(\Sigma(\mathcal{A})) & \simeq \mathbb{K}(\mathcal{A})[1], & \mathbb{K}_{*+1}(\Sigma(\mathcal{A})) & \simeq \mathbb{K}_{*}(\mathcal{A}), \\
\mathrm{THH}(\Sigma(\mathcal{A})) & \simeq \mathrm{THH}(\mathcal{A})[1], & \mathrm{THH}_{*+1}(\Sigma(\mathcal{A})) & \simeq \mathrm{THH}_{*}(\mathcal{A}), \\
\mathrm{TC}(\Sigma(\mathcal{A})) & \simeq \mathrm{TC}(\mathcal{A})[1], & \mathrm{TC}_{*+1}(\Sigma(\mathcal{A})) & \simeq \mathrm{TC}_{*}(\mathcal{A}) .
\end{aligned}
$$

Negative cyclic homology $\mathrm{HC}^{-}$and periodic cyclic homology HP are not examples of localizing invariants since they do not preserve filtered (homotopy) colimits. Nevertheless, as explained in [6], Examples 8.10 and 8.11, they factor through Mot $_{\mathrm{dg}}^{\text {loc }}$ thus giving rise to realizations. We obtain then the canonical isomorphisms:

$$
\begin{aligned}
\mathrm{HC}^{-}(\Sigma(\mathcal{A})) & \simeq \mathrm{HC}^{-}(\mathcal{A})[1], & \mathrm{HC}_{*+1}^{-}(\Sigma(\mathcal{A})) & \simeq \mathrm{HC}_{*}^{-}(\mathcal{A}), \\
\operatorname{HP}(\Sigma(\mathcal{A})) & \simeq \mathrm{HP}(\mathcal{A})[1], & \operatorname{HP}_{*+1}(\Sigma(\mathcal{A})) & \simeq \mathrm{HP}_{*}(\mathcal{A}) .
\end{aligned}
$$

Note that since HP is 2-periodic, the homologies of $\Sigma(\mathcal{A})$ and $\mathcal{A}$ can be obtained from each other by simply switching the degrees. To the best of the author's knowledge the isomorphisms (1.3)-(1.9) are new. They show us that $\Sigma(\mathcal{A})$ is a simple model for the suspension in all these classical invariants. ${ }^{2}$

We would like to mention that Kassel constructed an isomorphism related to (1.4), but for ordinary algebras over a field and with cyclic homology replaced by bivariant cyclic cohomology; see [12], Theorem 3.1. Instead of $\Gamma$, he considered the larger algebra of infinite matrices which have finitely many non-zero entries in each line and column.

Now let $X$ a quasi-compact and quasi-separated scheme. It is well known that the category of perfect complexes in the (unbounded) derived category of quasi-coherent sheaves on $X$ admits a dg-enhancement perf ${ }_{\mathrm{dg}}(X)$; see for instance [2], [20] or [6], Example 4.5. Due to [1], Theorem 1.3, [13], §5.2, and [22], §8, Theorem 5, the algebraic K-theory and the (topological) cyclic homology ${ }^{3}$ of the scheme $X$ can be obtained from the dg category perf $f_{\mathrm{dg}}(X)$ by applying the corresponding invariant. Therefore, when $\mathcal{A}=\operatorname{perf}_{\mathrm{dg}}(X)$, the above isomorphisms (1.3)-(1.9) suggest that the dg category $\Sigma\left(\right.$ perf $\left._{\mathrm{dg}}(X)\right)$ should be considered as the "noncommutative suspension"

\footnotetext{
${ }^{2}$ Recall that all these invariants take values in arbitrary degrees.

${ }^{3}$ In fact we can consider any variant of (topological) cyclic homology.
} 
or "noncommutative delooping" of the scheme $X$. This will be the subject of future research.

Acknowledgments. Theorem 1.2 answers affirmatively a question raised by Maxim Kontsevich in my Ph.D. thesis defense [26]. I deeply thank him for his insight. I am also grateful to Bernhard Keller, Marco Schlichting and Bertrand Toën for useful conversations and/or references.

Convention. Throughout the article $k$ will denote a commutative base ring with unit 1 . Given a dg algebra $H$ we will denote by $\underline{H}$ the dg category with a single object $*$ and with $H$ as the dg algebra of endomorphisms.

\section{Background on dg categories}

In this section we collect some notions and results on dg categories which will be used throughout the article.

Let $\ell(k)$ be the category of (unbounded) complexes of $k$-modules; we use cohomological notation. A differential graded (dg) category is a category enriched over $\mathcal{C}(k)$ and a $d g$ functor is a functor enriched over $\mathcal{C}(k)$; consult Keller's ICM address [16] for a survey on dg categories. The category of dg categories will be denoted by dgcat.

Notation 2.1. Let $\mathcal{A}$ be a dg category. The category $Z^{0}(\mathcal{A})$ has the same objects as $\mathcal{A}$ and morphisms given by $\mathrm{Z}^{0}(\mathcal{A})(x, y):=\mathrm{Z}^{0}(\mathcal{A}(x, y))$. The category $\mathrm{H}^{0}(\mathcal{A})$ has the same objects as $\mathcal{A}$ and morphisms given by $\mathrm{H}^{0}(\mathcal{A})(x, y):=\mathrm{H}^{0}(\mathcal{A}(x, y))$. The opposite dg category $\mathcal{A}^{\mathrm{op}}$ of $\mathcal{A}$ has the same objects as $\mathcal{A}$ and complexes of morphisms given by $\mathcal{A}^{\mathrm{op}}(x, y):=\mathcal{A}(y, x)$.

2.1. (Bi)modules. Let $\mathcal{A}$ be a dg category. A right $\mathcal{A}$-module $M$ is a $\mathrm{dg}$ functor $M: \mathcal{A}^{\mathrm{op}} \rightarrow \mathcal{C}_{\mathrm{dg}}(k)$ with values in the dg category $\mathcal{C}_{\mathrm{dg}}(k)$ of complexes of $k$-modules. We will denote by $\mathscr{C}(\mathcal{A})$ the category of right $\mathcal{A}$-modules; see [16], §2.3. As explained in [16], §3.1, the differential graded structure of $\mathcal{C}_{\mathrm{dg}}(k)$ makes $\mathcal{C}(\mathcal{A})$ naturally into

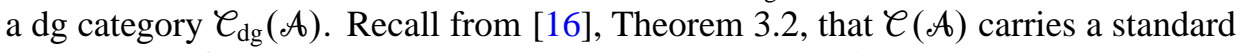
projective $\mathcal{C}(k)$-model structure. The derived category $\mathscr{D}(\mathcal{A})$ of $\mathcal{A}$ is the localization of $\mathcal{C}(\mathcal{A})$ with respect to the class of objectwise quasi-isomorphisms.

Notation 2.2. We denote by $\operatorname{perf}(\mathcal{A})$, resp. by $\operatorname{perf}_{\mathrm{dg}}(\mathcal{A})$, the full subcategory of $\mathcal{C}(\mathcal{A})$, resp. full dg subcategory of $\mathcal{C}_{\mathrm{dg}}(\mathcal{A})$, whose objects are the cofibrant right $\mathcal{A}$ modules that are compact ([21], Definition 4.2.7) in the triangulated category $\mathscr{D}(\mathcal{A})$.

Given dg categories $\mathcal{A}$ and $\mathscr{B}$ their tensor product $\mathcal{A} \otimes \mathscr{B}$ is defined as follows: the set of objects is the cartesian product and given objects $(x, z)$ and $(y, w)$ in $\mathscr{A} \otimes \mathscr{B}$, we set $(\mathcal{A} \otimes \mathscr{B})((x, z),(y, w)):=\mathcal{A}(x, y) \otimes \mathscr{B}(z, w)$. An $\mathcal{A}$ - $\mathscr{B}$-bimodule $X$ is a dg functor $X: \mathscr{A}^{\mathrm{op}} \otimes \mathscr{B} \rightarrow \mathcal{C}_{\mathrm{dg}}(k)$, i.e., a right $\mathscr{A}^{\mathrm{op}} \otimes \mathscr{B}$-module. 
2.2. Derived Morita equivalences. A dg functor $F: \mathcal{A} \rightarrow \mathscr{B}$ is a called a derived Morita equivalence if its derived extension of scalars functor $\mathbb{L} F_{!}: D(\mathcal{A}) \stackrel{\sim}{\longrightarrow} D(\mathscr{B})$ (see [31], §3) is an equivalence of triangulated categories. Due to [25], Theorem 5.3 (and [27]), the category dgcat carries a (cofibrantly generated) Quillen model structure whose weak equivalences are the derived Morita equivalences. We denote by Hmo the homotopy category hence obtained.

The tensor product of dg categories can be derived into a bifunctor $-\otimes^{\mathbb{L}}-$ on Hmo. Moreover, due to [31], Theorem 6.1, the bifunctor $-\otimes^{\mathbb{L}}-$ admits an internal Hom-functor rep $(-,-) .{ }^{4}$ Given $\mathrm{dg}$ categories $\mathcal{A}$ and $\mathscr{B}, \operatorname{rep}(\mathcal{A}, \mathscr{B})$ is the full $\mathrm{dg}$ subcategory of $\mathcal{C}_{\mathrm{dg}}\left(\mathscr{A}^{\mathrm{op}} \otimes^{\mathbb{L}} \mathscr{B}\right)$ spanned by the cofibrant $\mathscr{A}$ - $\mathscr{B}$-bimodules $X$ such that, for every object $x$ in $\mathcal{A}$, the right $\mathcal{B}$-module $X(x,-)$ is compact in $\mathscr{D}(\mathscr{B})$. The set of morphisms in Hmo from $\mathcal{A}$ to $\mathscr{B}$ is given by the set of isomorphism classes of the triangulated category $\mathrm{H}^{0}(\operatorname{rep}(\mathcal{A}, \mathscr{B}))$.

2.3. Exact sequences. A sequence of triangulated categories

$$
0 \rightarrow \mathcal{R} \stackrel{I}{\rightarrow} S \stackrel{P}{\rightarrow} \mathcal{T} \rightarrow 0
$$

is called exact if the composition is zero, the functor $I$ is fully faithful and the induced functor from the Verdier quotient $S / \mathcal{R}$ to $\mathcal{T}$ is cofinal, i.e., it is fully faithful and every object in $\mathcal{T}$ is a direct summand of an object of $S / \mathcal{R}$; see [21], §2. A sequence

$$
0 \rightarrow \mathcal{A} \stackrel{X}{\rightarrow} \mathscr{B} \stackrel{Y}{\rightarrow} \ell \rightarrow 0
$$

in $\mathrm{Hmo}$ is called exact if the induced sequence of triangulated categories

$$
0 \rightarrow \mathscr{D}(\mathcal{A}) \stackrel{-\otimes_{\mathcal{A}}^{\mathbb{Q}} X}{\longrightarrow} \mathscr{D}(\mathscr{B}) \stackrel{-\otimes_{\mathcal{B}}^{\square} Y}{\longrightarrow} \mathscr{D}(\mathcal{C}) \rightarrow 0
$$

is exact; see [16], §4.6.

\section{Infinite matrix algebras}

In this section we introduce the matrix algebras used in the construction of the universal suspension.

Definition 3.1. Given $n \in \mathbb{N}$, we denote by $\mathrm{M}_{n}$ the $k$-algebra of $n \times n$-matrices with coefficients in $k$. Let

$$
\mathrm{M}_{\infty}:=\bigcup_{n=1}^{\infty} \mathrm{M}_{n}
$$

\footnotetext{
${ }^{4}$ Denoted by $\mathbb{R} \underline{\text { Hom }}(-,-)$ in loc. cit.
} 
be the $k$-algebra of finite matrices, where $\mathrm{M}_{n} \subset \mathrm{M}_{n+1}$ via the map

$$
A \mapsto\left[\begin{array}{cc}
A & 0 \\
0 & 0
\end{array}\right]
$$

Note that $\mathrm{M}_{\infty}$ does not have a unit object. Moreover, transposition of matrices gives rise to an isomorphism of $k$-algebras

$$
(-)^{T}:\left(\mathrm{M}_{\infty}\right)^{\mathrm{op}} \stackrel{\sim}{\longrightarrow} \mathrm{M}_{\infty} .
$$

Notation 3.3. Given $k, l \in \mathbb{N}$, we denote by $E_{k l} \in \mathrm{M}_{\infty}$ the matrix

$$
\left(E_{k l}\right)_{i, j}:= \begin{cases}1 & \text { if } i=k \text { and } j=l \\ 0 & \text { otherwise }\end{cases}
$$

Note that given $k, l, m, n \in \mathbb{N}$, the product $E_{k l} \cdot E_{n m}$ equals $E_{k m}$ if $l=n$ and is zero otherwise. Given a non-negative integer $n \geq 0$, we denote by $I_{n} \in \mathrm{M}_{\infty}$ the matrix

$$
\left(I_{n}\right)_{i, j}:= \begin{cases}\mathbf{1} & \text { if } i=j \leq n \\ 0 & \text { otherwise }\end{cases}
$$

In particular, $I_{0}$ stands for the zero matrix.

Lemma 3.4. The $k$-algebra $\mathrm{M}_{\infty}$ has idempotent local units, i.e., for each finite family $A_{s}, s \in S$, of elements in $\mathrm{M}_{\infty}$ there exists an idempotent $E \in \mathrm{M}_{\infty}$ such that $E \cdot A_{s}=A_{s} \cdot E=A_{s}$ for all $s \in S$.

Proof. Since the matrices $A_{s}, s \in S$, have only a finite number of non-zero entries there exist natural numbers $m_{s}, s \in S$, such that $\left(A_{s}\right)_{i, j}=0$ when $i$ or $j$ is greater than $m_{s}$. Let $m:=\max \left\{m_{s} \mid s \in S\right\}$. If $E$ is the idempotent matrix $I_{m}$ we observe that $I_{m} \cdot A_{s}=A_{s} \cdot I_{m}=A_{s}$ for all $s \in S$.

Definition 3.5. Let $\Gamma$ be the $k$-algebra of $(\mathbb{N} \times \mathbb{N})$-matrices $A$ with coefficients in $k$ and satisfying the following two conditions:

(1) the set $\left\{A_{i, j} \mid i, j \in \mathbb{N}\right\}$ is finite;

(2) there exists a natural number $n_{A}$ (which depends on $A$ ) such that each row and each column has at most $n_{A}$ non-zero entries.

The $k$-module structure is defined entrywise and the multiplication is given by the ordinary matrix multiplication law; note that if $A, B \in \Gamma$ we can take $n_{A} \times n_{B}$ as the natural number $n_{A \cdot B}$. In contrast to $\mathrm{M}_{\infty}, \Gamma$ does have a unit object

$$
I_{i, j}:= \begin{cases}1 & \text { if } i=j \\ 0 & \text { otherwise }\end{cases}
$$


Moreover, transposition of matrices induces an isomorphism of $k$-algebras

$$
(-)^{T}: \Gamma^{\mathrm{op}} \stackrel{\sim}{\longrightarrow} \Gamma
$$

which extends isomorphism (3.2).

Now let us fix a bijection

$$
\theta: \mathbb{N} \stackrel{\sim}{\longrightarrow} \mathbb{N} \times \mathbb{N}, \quad n \mapsto\left(\theta_{1}(n), \theta_{2}(n)\right) ;
$$

take for instance the inverse of Cantor's classical pairing function. As in [23], Lemma 19, we define a $k$-algebra homomorphism

$$
\phi: \Gamma \rightarrow \Gamma, \quad A \mapsto \phi(A),
$$

by

$$
\phi(A)_{i, j}:= \begin{cases}A_{\theta_{1}(i), \theta_{1}(j)} & \text { if } \theta_{2}(i)=\theta_{2}(j), \\ 0 & \text { otherwise. }\end{cases}
$$

Note that the non-zero elements in line $i$, resp. in column $j$, of the matrix $\phi(A)$ are precisely the non-zero elements in line $i$, resp. in column $j$, of the matrix $A$.

Definition 3.6. Let $W$ be the $\Gamma$ - $\Gamma$-bimodule, which is $\Gamma$ as a left $\Gamma$-module, and whose right $\Gamma$-action is given by

$$
\Gamma \times \Gamma \rightarrow \Gamma, \quad(B, A) \mapsto B \cdot \phi(A) .
$$

Lemma 3.7. There exists a natural $\Gamma$ - $\Gamma$-bimodule isomorphism $\Gamma \oplus W \stackrel{\sim}{\longrightarrow} W$.

Proof. Consider the elements

$$
\alpha_{i, j}:= \begin{cases}\mathbf{1} & \text { if } \theta(j)=(i, 0), \\ 0 & \text { otherwise }\end{cases}
$$

and

$$
\beta_{i, j}:= \begin{cases}\mathbf{1} & \text { if } \theta(j)=\theta(i)+(0,1), \\ 0 & \text { otherwise }\end{cases}
$$

in $\Gamma$. Using $\alpha$ and $\beta$, we define maps

$$
\begin{array}{rlrl}
\Gamma \oplus W & \rightarrow W, & (A, B) & \mapsto A \cdot \alpha+B \cdot \beta, \\
W & \rightarrow \Gamma \oplus W, \quad B & \mapsto\left(B \cdot \alpha^{T}, B \cdot \beta^{T}\right) .
\end{array}
$$

The map (3.8) is a left $\Gamma$-module homomorphism. The fact that it is also a right $\Gamma$-module homomorphism follows from the equalities

$$
\beta \cdot \alpha^{T}=0, \quad \alpha \cdot \alpha^{T}=\beta \cdot \beta^{T}=I, \quad \alpha^{T} \cdot \alpha+\beta^{T} \cdot \beta=I .
$$


Moreover, since for every $A \in \Gamma$ we have

$$
A \cdot \alpha=\alpha \cdot \phi(A) \text { and } \phi(A) \cdot \beta=\beta \cdot \phi(A),
$$

we conclude that the maps (3.8) and (3.9) are inverse of each other.

Notation 3.10. Clearly the $k$-algebra $\mathrm{M}_{\infty}$ forms a two-sided ideal in $\Gamma$. We denote by $\Sigma$ the associated quotient $k$-algebra $\Gamma / \mathrm{M}_{\infty}$.

Alternatively, we can describe the quotient $k$-algebra $\Sigma$ as follows.

Proposition 3.11. The matrices

$$
\overline{I_{n}}:=I-I_{n}
$$

(see Notation 3.3) form a left denominator set $S$ in $\Gamma$ ([19], §4), i.e., $I \in S$, $S \cdot S \subset S$ and

(i) given $\overline{I_{n}} \in S$ and $E \in \Gamma$, there are $\overline{I_{m}} \in S$ and $E^{\prime} \in \Gamma$ such that $E^{\prime} \cdot \overline{I_{n}}=\overline{I_{m}} \cdot E$;

(ii) if $\overline{I_{n}} \in S$ and $E \in \Gamma$ satisfy $E \cdot \overline{I_{n}}=0$, there is $\overline{I_{m}} \in S$ such that $\overline{I_{m}} \cdot E=0$.

Moreover, the localized $k$-algebra $\Gamma\left[S^{-1}\right]^{5}$ is naturally isomorphic to $\Sigma$.

Proof. In order to simplify the proof we consider the following block-matrix graphical notation

$$
E=k\left[\begin{array}{c|c}
E_{a} & E_{b} \\
\hline E_{c} & E_{d}
\end{array}\right] \in \Gamma,
$$

where $k, l \in \mathbb{N}, E_{a}$ is a $(k \times l)$-matrix, $E_{b}$ is a $(k \times \mathbb{N})$-matrix, $E_{c}$ is a $(\mathbb{N} \times l)$-matrix, and $E_{d}$ is a $(\mathbb{N} \times \mathbb{N})$-matrix. Under this notation we have, for $n \in \mathbb{N}$, the equalities

$$
\overline{I_{n}} \cdot n\left[\begin{array}{c|c}
E_{a} & E_{b} \\
\hline E_{c} & E_{d}
\end{array}\right]=n\left[\begin{array}{c|c}
0 & 0 \\
\hline E_{c} & E_{d}
\end{array}\right]
$$

and

$$
n\left[\begin{array}{c|c}
E_{a} & E_{b} \\
\hline E_{c} & E_{d}
\end{array}\right] \cdot \overline{I_{n}}=n\left[\begin{array}{l|l}
0 & E_{b} \\
\hline 0 & E_{d}
\end{array}\right] .
$$

By definition $I=\overline{I_{0}} \in S$. Equalities (3.12) and (3.13), and the fact that $\overline{I_{0}}=I$, imply that

$$
\overline{I_{n}} \cdot \overline{I_{m}}=\overline{I_{\max \{n, m\}}}, \quad n \geq 0 .
$$

This shows that $S \cdot S \subset S$.

${ }^{5}$ Since $S$ is a left denominator set, this $k$-algebra is given by left fractions, i.e., equivalence classes of pairs $\left(\overline{I_{n}}, E\right)$ modulo the relation which identifies $\left(\overline{I_{n}}, E\right)$ with $\left(\overline{I_{m}}, E^{\prime}\right)$ if there are $B, B^{\prime} \in \Gamma$ such that $B \cdot \overline{I_{n}}=B^{\prime} \cdot \overline{I_{m}}$ belongs to $S$ and $B \cdot E=B^{\prime} \cdot E^{\prime}$. 
(i) Note first that when $n=0$ the claim is trivial. Since $E$ belongs to $\Gamma$, there exist natural numbers $m_{j}, 1 \leq j \leq n$, such that $E_{i, j}=0$ for $i \geq m_{j}$ and $1 \leq j \leq n$. Take $m=\max \left\{n, m_{j} \mid 1 \leq j \leq n\right\}$. Then, we have the equality

$$
\overline{I_{m}} \cdot m\left[\begin{array}{c|c}
E_{a} & E_{b} \\
\hline E_{c} & E_{d}
\end{array}\right]=m\left[\begin{array}{c|c}
0 & 0 \\
\hline 0 & E_{d}
\end{array}\right] .
$$

Since $m \geq n$, the above equality (3.13) shows us that we can take for $E^{\prime}$ the above matrix (3.14). This proves the claim.

(ii) When $n=0$ the claim is trivial. If $E \cdot \overline{I_{n}}=0$ the above equality (3.13) shows us that

$$
E=n\left[\begin{array}{c|c}
E_{a} & 0 \\
\hline E_{c} & 0
\end{array}\right] .
$$

Since $E$ belongs to $\Gamma$, there exist natural numbers $m_{j}, 1 \leq j \leq n$, such that $E_{i, j}=0$ for $i \geq m_{j}$ and $1 \leq j \leq n$. Take $m=\max \left\{m_{j} \mid 1 \leq j \leq n\right\}$. Then the above description (3.15) combined with equality (3.13) show us that $\bar{I}_{m} \cdot E=0$. This proves the claim.

We now show that the localized $k$-algebra $\Gamma\left[S^{-1}\right]$ is naturally isomorphic to $\Sigma$. Since the matrices

$$
I_{n}=I-\overline{I_{n}}, \quad n \geq 0,
$$

belong to $\mathrm{M}_{\infty}$, we conclude that all the elements of the set $S$ become the identity object in $\Sigma$. Therefore, by the universal property of $\Gamma\left[S^{-1}\right]$ we obtain a $k$-algebra map

$$
\Gamma\left[S^{-1}\right] \rightarrow \Sigma \text {. }
$$

On the other hand, the kernel of the localization map $\Gamma \rightarrow \Gamma\left[S^{-1}\right]$ consists of those matrices $E \in \Gamma$ for which $\overline{I_{n}} \cdot E=0$ for some $n \geq 0$. Due to equality (3.12) we observe that the elements of $\mathrm{M}_{\infty}$ satisfy this condition. Therefore, by the universal property of $\Sigma:=\Gamma / \mathrm{M}_{\infty}$ we obtain a $k$-algebra map

$$
\Sigma \rightarrow \Gamma\left[S^{-1}\right] \text {. }
$$

The maps (3.16) and (3.17) are clearly inverse of each other and so the proof is finished.

Lemma 3.18. The algebras $\mathrm{M}_{\infty}, \Gamma$ and $\Sigma$ are flat as $k$-modules.

Proof. We start by proving this proposition in the particular case where the base ring $k$ is $\mathbb{Z}$. In this case the underlying $\mathbb{Z}$-modules of $\left(\mathrm{M}_{\infty}\right)_{\mathbb{Z}}$ and $\Gamma_{\mathbb{Z}}$ are torsion-free and so by [33], Corollary 3.1.5, are flat. Due to Proposition 3.11, $\Sigma_{\mathbb{Z}}$ identifies with the (left) localization of $\Gamma_{\mathbb{Z}}$ with respect to the set $S$, and so a standard argument shows that the right $\Gamma_{\mathbb{Z}}$-module $\Sigma_{\mathbb{Z}}$ is flat. Since $\Gamma_{\mathbb{Z}}$ is flat as a $\mathbb{Z}$-module, we conclude that $\Sigma_{\mathbb{Z}}$ is also flat as a $\mathbb{Z}$-module. 
Let us now consider the general case. Clearly we have a natural isomorphism of $k$-modules

$$
\left(\mathrm{M}_{\infty}\right)_{\mathbb{Z}} \otimes_{\mathbb{Z}} k \stackrel{\sim}{\longrightarrow}\left(\mathrm{M}_{\infty}\right)_{k} .
$$

By [4], Lemma 4.7.1, we also have natural isomorphisms of $k$-modules

$$
\Gamma_{\mathbb{Z}} \otimes_{\mathbb{Z}} k \stackrel{\sim}{\longrightarrow} \Gamma_{k} \quad \text { and } \quad \Sigma_{\mathbb{Z}} \otimes_{\mathbb{Z}} k \stackrel{\sim}{\longrightarrow} \Sigma_{k} .
$$

Therefore, since flat modules are stable under extension of scalars, the proof is achieved.

\section{An exact sequence}

Let $H$ be a $k$-algebra and $J \subset H$ a two-sided ideal.

Definition 4.1. The category $\&$ of idempotents of $J$ is defined as follows: its objects are the symbols $\boldsymbol{u}$, where $u$ is an idempotent of $J$; the $k$-module $\mathcal{L}\left(\boldsymbol{u}, \boldsymbol{u}^{\prime}\right)$ of morphisms from $\boldsymbol{u}$ to $\boldsymbol{u}^{\prime}$ is $u J u^{\prime}$; composition is given by multiplication in $J$ and the unit of each object $\boldsymbol{u}$ is the idempotent $u$. Associated to $H$ and $J$ there is also a $\mathcal{g}$ - $\underline{H}$-bimodule $X$ such that $X(\boldsymbol{u}, *):=u J$, with left and right actions given by multiplication.

Recall from [14], Example 3.3 (b), that if $H$ and $J$ are flat as $k$-modules and $J$ has idempotent local units (i.e., for each finite family $a_{s}, s \in S$, of elements in $J$ there exists an idempotent $u \in J$ such that $u a_{s}=a_{s} u=a_{s}$ for all $s \in S$ ) we have an exact sequence

$$
0 \rightarrow g \stackrel{X}{\rightarrow} \underline{H} \rightarrow \underline{H / J} \rightarrow 0 .
$$

in Hmo. By Lemmas 3.4 and 3.18, if we take $H=\Gamma$ and $J=\mathrm{M}_{\infty}$, we obtain the exact sequence

$$
0 \rightarrow \mathcal{M}_{\infty} \stackrel{X}{\rightarrow} \underline{\Gamma} \rightarrow \underline{\Sigma} \rightarrow 0
$$

in $\mathrm{Hmo}$.

Proposition 4.3. The dg functor

$$
\underline{k} \rightarrow \mathcal{M}_{\infty}, \quad * \mapsto \boldsymbol{E}_{\mathbf{1 1}}
$$

(see Notation 3.3), is a derived Morita equivalence.

Proof. We will prove a stronger statement, namely that the above functor (4.4) is a Morita equivalence; see [24], §2. The category $\mathcal{M}_{\infty}$ is by definition enriched over $k$-modules and the classical theory of Morita holds in this setting. Let Mod- $\mathcal{M}_{\infty}$ be the abelian category of right $\mathcal{M}_{\infty}$-modules (i.e., contravariant $k$-linear functors from $\mathcal{M}_{\infty}$ to $k$-modules) and

$$
\widehat{(-)}: \mathcal{M}_{\infty} \rightarrow \operatorname{Mod}-\mathcal{M}_{\infty}, \quad \boldsymbol{E} \mapsto \mathcal{M}_{\infty}(-, \boldsymbol{E})=: \widehat{\boldsymbol{E}},
$$


the (enriched) Yoneda functor. Following [24], Theorems 2.2 and 2.5, we need to show that $\widehat{\boldsymbol{E}_{11}}$ is a small projective generator of Mod- $\mathcal{M}_{\infty}$ and that its ring of endomorphisms is isomorphic to $k$. Note that we have natural isomorphisms

$$
\operatorname{Hom}_{\operatorname{Mod}-\mathcal{M}_{\infty}}\left(\widehat{\boldsymbol{E}_{11}}, \widehat{\boldsymbol{E}_{11}}\right) \simeq \mathcal{M}_{\infty}\left(\boldsymbol{E}_{\mathbf{1 1}}, \boldsymbol{E}_{\mathbf{1 1}}\right)=E_{11} \cdot \mathrm{M}_{\infty} \cdot E_{11} \simeq k .
$$

Moreover, $\widehat{\boldsymbol{E}_{11}}$ is small and projective by definition. Therefore, it only remains to show that $\widehat{\boldsymbol{E}_{\mathbf{1 1}}}$ is a generator, i.e., , that every right $\mathcal{M}_{\infty}$-module $P$ is an epimorphic image of a sum of (possibly infinitely many) copies of $\widehat{\boldsymbol{E}_{\mathbf{1 1}}}$. Given an object $\boldsymbol{E}$ in $\mathcal{M}_{\infty}$ we have, by the (enriched) Yoneda lemma, an isomorphism

$$
\operatorname{Hom}_{\text {Mod- }-\mathcal{M}_{\infty}}(\widehat{\boldsymbol{E}}, P) \simeq P(\boldsymbol{E})
$$

and so we obtain a natural epimorphism

$$
\underset{\boldsymbol{E} \in \mathcal{M}_{\infty}}{\bigoplus} \underset{P(\boldsymbol{E})}{\bigoplus} \hat{\boldsymbol{E}} \rightarrow P
$$

This shows that it suffices to treat the case where $P$ is of shape $\hat{\boldsymbol{E}}$. We consider first the cases $\boldsymbol{E}=\mathbf{E}_{\boldsymbol{n} \boldsymbol{n}}, \boldsymbol{n} \in \mathbb{N}$. The following morphisms in $\mathcal{M}_{\infty}$

$$
\boldsymbol{E}_{\mathbf{1 1}} \stackrel{E_{11} \cdot E_{1 n} \cdot E_{n n}}{\longrightarrow} \boldsymbol{E}_{\boldsymbol{n} n} \text { and } \boldsymbol{E}_{\boldsymbol{n} \boldsymbol{n}} \stackrel{E_{n n} \cdot E_{n 1} \cdot E_{11}}{\longrightarrow} \boldsymbol{E}_{\mathbf{1 1}}
$$

show us that $\boldsymbol{E}_{\mathbf{1 1}}$ and $\boldsymbol{E}_{\boldsymbol{n} \boldsymbol{n}}$ are isomorphic and so the claim follows.

We consider now the cases $\boldsymbol{E}=\boldsymbol{I}_{\boldsymbol{m}}, m \in \mathbb{N}$. The natural morphisms in $\mathcal{M}_{\infty}$

$$
\boldsymbol{E}_{\boldsymbol{n} \boldsymbol{n}} \stackrel{E_{n n} \cdot E_{n n} \cdot I_{m}}{\longrightarrow} \boldsymbol{I}_{\boldsymbol{m}}, \quad 1 \leq n \leq m,
$$

give rise to a map

$$
\bigoplus_{n \geq 1}^{m} \widehat{\boldsymbol{E}_{\boldsymbol{n}} \boldsymbol{n}} \rightarrow \widehat{\boldsymbol{I}_{\boldsymbol{m}}}
$$

in Mod- $\mathcal{M}_{\infty}$. In order to show that the map (4.5) is surjective, we need to show that its evaluation

$$
\bigoplus_{n \geq 1}^{m} \mathcal{M}_{\infty}\left(\boldsymbol{B}, \boldsymbol{E}_{\boldsymbol{n} n}\right) \rightarrow \mathcal{M}_{\infty}\left(\boldsymbol{B}, \boldsymbol{I}_{\boldsymbol{m}}\right)
$$

at each object $\boldsymbol{B}$ of $\mathcal{M}_{\infty}$ is surjective. We have $I_{m}=\sum_{n \geq 1}^{m} E_{n n}$, and so (4.6) identifies with the natural map

$$
\bigoplus_{n \geq 1}^{m}\left(B \cdot \mathrm{M}_{\infty} \cdot E_{n n}\right) \rightarrow B \cdot \mathrm{M}_{\infty} \cdot\left(\sum_{n \geq 1}^{m} E_{n n}\right),
$$

which is easily seen to be surjective. Since $\boldsymbol{E}_{\mathbf{1 1}}$ is isomorphic to $\boldsymbol{E}_{\boldsymbol{n} \boldsymbol{n}}, n \in \mathbb{N}$, the claim is proved. 
Finally, we consider the case of a general object $\boldsymbol{E}$ in $\mathcal{M}_{\infty}$. Since $E$ has only a finite number of non-zero entries there exists a natural number $m$ such that $E_{i, j}=0$ when $i$ or $j$ is greater than $m$. We have then the equality

$$
E \cdot I_{m}=I_{m} \cdot E=E .
$$

This implies that the composition

$$
\boldsymbol{E} \stackrel{E \cdot I_{m} \cdot I_{m}}{\longrightarrow} I_{\boldsymbol{m}} \stackrel{I_{m} \cdot I_{m} \cdot E}{\longrightarrow} \boldsymbol{E}
$$

equals the identity map of the object $\boldsymbol{E}$. Since the abelian category Mod- $\mathcal{M}_{\infty}$ is idempotent complete, we conclude that the right $\mathcal{M}_{\infty}$-module $\widehat{\boldsymbol{E}}$ is a direct factor of $\widehat{\boldsymbol{I}_{\boldsymbol{m}}}$. This achieves the proof.

By combining the exact sequence (4.2) with the derived Morita equivalence (4.4) we obtain in $\mathrm{Hmo}$ an exact sequence

$$
0 \rightarrow \underline{k} \rightarrow \underline{\Gamma} \rightarrow \underline{\Sigma} \rightarrow 0 .
$$

Notation 4.8. Given a dg category $\mathcal{A}$, we denote by $\Gamma(\mathcal{A})$ the dg category $\underline{\Gamma} \otimes \mathcal{A}$ and by $\Sigma(\mathcal{A})$ the dg category $\underline{\Sigma} \otimes \mathcal{A}$; see $\S 2.1$.

Proposition 4.9. For every dg category $\mathcal{A}$ we have an exact sequence

$$
0 \rightarrow \mathcal{A} \rightarrow \Gamma(\mathcal{A}) \rightarrow \Sigma(\mathcal{A}) \rightarrow 0
$$

in $\mathrm{Hmo}$.

Proof. The exact sequence (4.7) and [7], Proposition 1.6.3, leads to the exact sequence

$$
0 \rightarrow \underline{k} \otimes^{\mathbb{L}} \mathcal{A} \rightarrow \underline{\Gamma} \otimes^{\mathbb{L}} \mathcal{A} \rightarrow \underline{\Sigma} \otimes^{\mathbb{L}} \mathcal{A} \rightarrow 0
$$

in Hmo. By Lemma 3.18 the algebras $\Gamma$ and $\Sigma$ are flat as $k$-modules and so the derived tensor products are identified in Hmo with the ordinary ones. Moreover, we have a natural isomorphism $\underline{k} \otimes^{\mathbb{L}} \mathcal{A} \simeq \mathcal{A}$.

\section{Flasqueness of $\Gamma$}

Definition 5.1. Let $\mathcal{A}$ be a dg category with sums (i.e., the diagonal dg functor $\Delta: \mathcal{A} \rightarrow \mathcal{A} \times \mathcal{A}$ admits a left adjoint $\oplus: \mathcal{A} \times \mathcal{A} \rightarrow \mathcal{A}$ ) such that $Z^{0}(\mathcal{A})$ is equivalent to $\operatorname{perf}(\mathcal{A})$; see Notations 2.1 and 2.2. Under these hypothesis, we say that $\mathcal{A}$ is flasque if there exists a dg functor $\tau: \mathcal{A} \rightarrow \mathcal{A}$ and a natural isomorphism $\operatorname{Id} \oplus \tau \simeq \tau$.

Proposition 5.2. The dg category perf $_{\mathrm{dg}}(\Gamma)$ is flasque. 
Proof. Notice first that since we have an isomorphism of $k$-algebras

$$
(-)^{T}: \Gamma^{\mathrm{op}} \stackrel{\sim}{\longrightarrow} \Gamma,
$$

it is equivalent to show that the dg category perf $f_{d g}\left(\Gamma^{o p}\right)$ is flasque. By definition, perf $_{\mathrm{dg}}\left(\Gamma^{\mathrm{op}}\right)$ has sums and $Z^{0}\left(\right.$ perf $\left._{\mathrm{dg}}\left(\Gamma^{\mathrm{op}}\right)\right)$ is equivalent to perf $\left(\Gamma^{\mathrm{op}}\right)$. Now recall from Definition 3.6 the construction of the $\Gamma$ - $\Gamma$-bimodule $W$. As explained in [16], $\S 3.8$, the bimodule $W$ gives rise to a Quillen adjunction

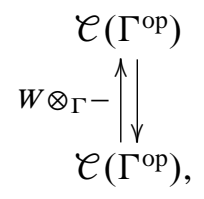

which is moreover compatible with the $\mathcal{C}(k)$-enrichment. Since the $\Gamma$ - $\Gamma$-bimodule $W$ is $\Gamma$ as a left $\Gamma$-module, the left Quillen dg functor

$$
W \otimes_{\Gamma}-: \ell_{\mathrm{dg}}\left(\Gamma^{\mathrm{op}}\right) \rightarrow \mathcal{\ell}_{\mathrm{dg}}\left(\Gamma^{\mathrm{op}}\right)
$$

restricts to a dg functor

$$
\tau: \operatorname{perf}_{\mathrm{dg}}\left(\Gamma^{\mathrm{op}}\right) \rightarrow \operatorname{perf}_{\mathrm{dg}}\left(\Gamma^{\mathrm{op}}\right) .
$$

Moreover, given an object $P$ in perf $\mathrm{dg}_{\mathrm{g}}\left(\Gamma^{\mathrm{op}}\right)$ we have a functorial isomorphism

$$
P \oplus \tau(P)=P \oplus\left(W \otimes_{\Gamma} P\right) \simeq(\Gamma \oplus W) \otimes_{\Gamma} P \underset{\sim}{\stackrel{\psi}{\sim}} W \otimes_{\Gamma} P=\tau(P),
$$

where $\psi$ is obtained by tensoring the $\Gamma$ - $\Gamma$-bimodule isomorphism $\Gamma \oplus W \stackrel{\sim}{\longrightarrow} W$ of Lemma 3.7 with $P$. This finishes the proof.

Lemma 5.3. Let $\mathcal{A}$ and $\mathscr{B}$ be two dg categories, with $\mathcal{A}$ flasque. Then the dg category $\operatorname{rep}(\mathscr{B}, \mathcal{A})($ see $\S 2.2)$ is also flasque.

Proof. By construction, the dg category $\operatorname{rep}(\mathscr{B}, \mathcal{A})$ has sums and $Z^{0}(\operatorname{rep}(\mathscr{A}, \mathscr{B}))$ is equivalent to $\operatorname{perf}(\operatorname{rep}(\mathcal{A}, \mathscr{B}))$. Moreover, since $\mathcal{A}$ is flasque and $\operatorname{rep}(\mathscr{B},-)$ is a 2 -functor which preserves (derived) products, we obtain a dg functor

$$
\operatorname{rep}(\mathscr{B}, \tau): \operatorname{rep}(\mathscr{B}, \mathcal{A}) \rightarrow \operatorname{rep}(\mathscr{B}, \mathcal{A})
$$

and a natural isomorphism $\operatorname{Id} \oplus \operatorname{rep}(\mathscr{B}, \tau) \simeq \operatorname{rep}(\mathscr{B}, \tau)$.

Let us now recall the definition of the algebraic K-theory of dg categories. Given a dg category $\mathcal{A}$ we denote by perf ${ }^{\mathfrak{W}}(\mathcal{A})$ the Waldhausen category perf $(\mathcal{A})$, whose weak equivalences and cofibrations are those of the Quillen model structure on $\mathcal{C}(\mathcal{A})$; see [8], §3. The algebraic K-theory spectrum $K(\mathscr{A})$ of $\mathcal{A}$ is the Waldhausen Ktheory spectrum ([32]) of perf ${ }^{\text {W }}(\mathcal{A})$. Given a dg functor $F: \mathcal{A} \rightarrow \mathscr{B}$, the extension 
of scalars left Quillen functor $F_{!}: \mathcal{C}(\mathcal{A}) \rightarrow \mathcal{C}(\mathscr{B})$ preserves weak equivalences, cofibrations, and pushouts. Therefore, it restricts to an exact functor $F_{!}:$perf ${ }^{W}(\mathcal{A}) \rightarrow$ perf ${ }^{\mathfrak{W}}(\mathscr{B})$ between Waldhausen categories and so it gives rise to a morphism of spectra $K(F): K(\mathcal{A}) \rightarrow K(\mathscr{B})$.

Lemma 5.4. Let A be a flasque dg category. Then its algebraic K-theory spectrum $K(\mathcal{A})$ is contractible.

Proof. By applying the functor $Z^{0}(-)$ to $\mathcal{A}$ and $\tau$, we obtain an exact functor

$$
Z^{0}(\tau): \operatorname{perf}^{w}(\mathcal{A}) \rightarrow \operatorname{perf}^{w}(\mathcal{A})
$$

and a natural isomorphism Id $\oplus Z^{0}(\tau) \simeq Z^{0}(\tau)$. Since Waldhausen's K-theory satisfies additivity ([32], Proposition 1.3.2(4)), we have the equality

$$
K(\mathrm{Id})+K\left(\mathrm{Z}^{0}(\tau)\right)=K\left(\mathrm{Z}^{0}(\tau)\right)
$$

in the homotopy category of spectra. Therefore, we conclude that $\operatorname{Id}_{K(\mathcal{A})} \simeq K(\mathrm{Id})$ is the trivial map. This shows that the algebraic K-theory spectrum $K(\mathcal{A})$ is contractible.

\section{Proof of Theorem 1.2}

We start by showing that $\Gamma(\mathcal{A})$ (see Notation 4.8) becomes the zero object in the triangulated category $\operatorname{Mot}_{\mathrm{dg}}^{\mathrm{loc}}(e)$ after application of $\mathcal{U}_{\mathrm{dg}}^{\mathrm{loc}}$. Since $\Gamma(\mathcal{A}) \simeq \Gamma^{\mathbb{L}} \mathcal{A}$ and $U_{\mathrm{dg}}^{\text {loc }}$ is symmetric monoidal with respect to a homotopy colimit preserving symmetric monoidal structure on $\mathrm{Mot}_{\mathrm{dg}}^{\mathrm{loc}}$ (see [6], Theorem 7.5), it suffices to show that $\underline{\Gamma}$ becomes the zero object in $\operatorname{Mot}_{\mathrm{dg}}^{\text {loc }}(e)$.

Recall from [28], §17, that the universal localizing invariant admits the factorization

$$
u_{\mathrm{dg}}^{\text {loc }}: \mathrm{HO}(\text { dgcat }) \stackrel{u_{\mathrm{dg}}^{\text {add }}}{\longrightarrow} \operatorname{Mot}_{\mathrm{dg}}^{\text {add }} \stackrel{\gamma}{\rightarrow} \operatorname{Mot}_{\mathrm{dg}}^{\mathrm{loc}},
$$

where Mot $_{\mathrm{dg}}^{\text {add }}$ is the additive motivator ${ }^{6}$ and $\gamma$ is a localizing morphism between triangulated derivators. Moreover, due to [5], Proposition 3.7, the objects $\mathcal{U}_{\mathrm{dg}}^{\text {add }}(\mathscr{B})[n]$, with $\mathscr{B}$ a dg cell and $n \in \mathbb{Z}$, form a set of (compact) generators of the triangulated category $\operatorname{Mot}_{\mathrm{dg}}^{\mathrm{add}}(e)$. Therefore, $\mathcal{U}_{\mathrm{dg}}^{\text {add }}(\underline{\Gamma})$ is the zero object in $\operatorname{Mot}_{\mathrm{dg}}^{\text {add }}(e)$ if and only the spectra of morphisms $\mathbb{R} \underline{\operatorname{Hom}}\left(\mathcal{U}_{\mathrm{dg}}^{\text {add }}(\mathcal{B}), \mathcal{U}_{\mathrm{dg}}^{\text {add }}(\underline{\Gamma})\right)$, with $\mathscr{B}$ a dg cell, are (homotopically) trivial; see [5], §A.3. By [28], Theorem 15.10, we have the equivalences

$$
\mathbb{R} \underline{\operatorname{Hom}}\left(U_{\mathrm{dg}}^{\mathrm{add}}(\mathscr{B}), U_{\mathrm{dg}}^{\mathrm{add}}(\underline{\Gamma})\right) \simeq K \operatorname{rep}(\mathscr{B}, \underline{\Gamma}) \simeq K \operatorname{rep}\left(\mathscr{B}, \operatorname{perf}_{\mathrm{dg}}(\underline{\Gamma})\right) .
$$

\footnotetext{
${ }^{6}$ The additive motivator has a construction similar to the localizing one. Instead of imposing localization we impose the weaker requirement of additivity.
} 
Therefore, Proposition 5.2 and Lemmas 5.3 and 5.4 imply that $\mathcal{U}_{\mathrm{dg}}^{\mathrm{add}}(\underline{\Gamma})$ is the zero object in $\operatorname{Mot}_{\mathrm{dg}}^{\text {add }}(e)$. From the above factorization (6.1) we conclude that $\mathcal{U}_{\mathrm{dg}}^{\text {loc }}(\underline{\Gamma})$ (and so $\left.\mathcal{U}_{\mathrm{dg}}^{\text {loc }}(\Gamma(\mathcal{A}))\right)$ is the zero object in $\operatorname{Mot}_{\mathrm{dg}}^{\text {loc }}(e)$.

Now recall from Proposition 4.9 that we have an exact sequence

$$
0 \rightarrow \mathcal{A} \rightarrow \Gamma(\mathcal{A}) \rightarrow \Sigma(\mathcal{A}) \rightarrow 0 .
$$

By applying the universal localizing invariant to the preceding exact sequence we obtain a distinguished triangle

$$
U_{\mathrm{dg}}^{\mathrm{loc}}(\mathcal{A}) \rightarrow u_{\mathrm{dg}}^{\mathrm{loc}}(\Gamma(\mathcal{A})) \rightarrow u_{\mathrm{dg}}^{\mathrm{loc}}(\Sigma(\mathcal{A})) \rightarrow \mathcal{U}_{\mathrm{dg}}^{\mathrm{loc}}(\mathcal{A})[1]
$$

in $\operatorname{Mot}_{\mathrm{dg}}^{\text {loc }}(e)$. Therefore, since $\mathcal{U}_{\mathrm{dg}}^{\text {loc }}(\Gamma(\mathcal{A}))$ is the zero object, we have a canonical isomorphism

$$
\mathcal{U}_{\mathrm{dg}}^{\mathrm{loc}}(\Sigma(\mathcal{A})) \stackrel{\sim}{\longrightarrow} U_{\mathrm{dg}}^{\mathrm{loc}}(\mathcal{A})[1] .
$$

\section{References}

[1] A. Blumberg and M. Mandell, Localization theorems in topological Hochschild homology and topological cyclic homology. Preprint 2008. arXiv:0802.3938

[2] A. I. Bondal and M. M. Kapranov, Enhanced triangulated categories. Mat. Sb. 181 (1990), 669-683; English transl. Math. USSR-Sb. 70 (1991), 93-107. Zbl 0719.18005 MR 1055981

[3] A. Bondal and M. Van den Bergh, Generators and representability of functors in commutative and noncommutative geometry. Moscow Math. J. 3 (2003), 1-36. Zbl 1135.18302 MR 1996800

[4] G. Cortiñas and A. Thom, Bivariant algebraic K-theory. J. Reine Angew. Math. 610 (2007), 71-123. Zbl 1152.19002 MR 2359851

[5] D.-C. Cisinski and G. Tabuada, Non-connective $K$-theory via universal invariants. Compositio Math. 147 (2011), 1281-1320. Zbl 05938398

[6] D.-C. Cisinski and G. Tabuada, Symmetric monoidal structure on noncommutative motives. J. K-Theory, to appear. Preprint 2010. arXiv:1001.0228

[7] V. Drinfeld, DG quotients of DG categories. J. Algebra 272 (2004), 643-691. Zbl 1064.18009 MR 2028075

[8] D. Dugger and B. Shipley, $K$-theory and derived equivalences. Duke Math. J. 124 (2004), 587-617. Zbl 1056.19002 MR 2085176

[9] A. Grothendieck, Les Dérivateurs. Manuscript 1990/1991. Available at http://www.math.jussieu.fr/ maltsin/groth/Derivateurs.html

[10] D. Kaledin, Motivic structures in noncommutative geometry. To appear in the Proceedings of the ICM 2010. arXiv:1003.3210

[11] M. Karoubi and O. Villamayor, $K$-théorie algébrique et $K$-théorie topologique I. Math. Scand. 28 (1971), 265-307 (1972). Zbl 0231.18018 MR 0313360 
[12] C. Kassel, Caractère de Chern bivariant. K-Theory 3 (1989), 367-400. Zbl 0701.18008 MR 1047193

[13] B. Keller, On the cyclic homology of ringed spaces and schemes. Doc. Math. 3 (1998), 231-259. Zbl 0917.19002 MR 1647519

[14] B. Keller, Invariance and localization for cyclic homology of DG algebras. J. Pure Appl. Algebra 123 (1998), 223-273. Zbl 0890.18007 MR 1492902

[15] B. Keller, On the cyclic homology of exact categories. J. Pure Appl. Algebra 136 (1999), 1-56. Zbl 0923.19004 MR 1667558

[16] B. Keller, On differential graded categories. In Proc. Internat. Congr. Mathematicians. Vol. II, European Math. Soc. Publ. House, Zürich 2006, 151-190. Zbl 1140.18008 MR 2275593

[17] M. Kontsevich, Non-commutative motives. Talk at the IAS on the occasion of the 61st birthday of Pierre Deligne, October 2005. Video available at http://video.ias.edu/Geometry-and-Arithmetic

[18] M. Kontsevich, Notes on motives in finite characteristic. In Algebra, arithmetic, and geometry: in honor of Yu. I. Manin. Vol. II, Progr. Math. 270, Birkhäuser, Boston 2009, 213-247. Zbl 05778256 MR 2641191

[19] T. Y. Lam, Lectures on modules and rings. Graduate Texts in Math. 189. Springer-Verlag, New York 1999. Zbl 0911.16001 MR 1653294

[20] V. A. Lunts and D. O. Orlov, Uniqueness of enhancement for triangulated categories. $J$. Amer. Math. Soc. 23 (2010), 853-908. Zbl 1197.14014 MR 2629991

[21] A. Neeman, Triangulated categories. Ann. of Math. Stud. 148. Princeton University Press, Princeton, NJ, 2001. Zbl 0974.18008 MR 1812507

[22] M. Schlichting, Negative $K$-theory of derived categories. Math. Z. 253 (2006), 97-134. Zbl 1090.19002 MR 2206639

[23] M. Schlichting, The Mayer-Vietoris principle for Grothendieck-Witt groups of schemes. Invent. Math. 179 (2010), 349-433. Zbl 1193.19005 MR 2570120

[24] S. Schwede, Morita theory in abelian, derived and stable model categories. In Structured ring spectra. London Math. Soc. Lecture Note Ser. 315, Cambridge University Press, Cambridge 2004, 33-86. Zbl 1088.16010 MR 2122154

[25] G. Tabuada, Invariants additifs de dg-catégories. Internat. Math. Res. Notices 2005 (2005), No. 53, 3309-3339. Zbl 1094.18006 MR 2196100

[26] G. Tabuada, Théorie homotopique des DG-catégories. Ph.D. thesis, Université Paris Diderot - Paris 7, Paris 2007. arXiv:0710.4303

[27] G. Tabuada, Corrections à "Invariants additifs de dg-catégories". Internat. Math. Res. Notices 2007 (2007), Article ID rnm149.

[28] G. Tabuada, Higher $K$-theory via universal invariants. Duke Math. J. 145 (2008), 121-206. Zbl 1166.18007 MR 2451292

[29] G. Tabuada, Generalized spectral categories, topological Hochschild homology and trace maps. Algebr. Geom. Topol. 10 (2010), 137-213. Zbl 1206.55012 MR 2580431

[30] R. W. Thomason and T. Trobaugh, Higher algebraic $K$-theory of schemes and of derived categories. In The Grothendieck Festschrift, Vol. III, Progr. Math. 88, Birkhäuser, Boston 1990, 247-436. Zbl 0731.14001 MR 1106918 
[31] B. Toën, The homotopy theory of $d g$-categories and derived Morita theory. Invent. Math. 167 (2007), 615-667. Zbl 1118.18010 MR 2276263

[32] F. Waldhausen, Algebraic $K$-theory of spaces. In Algebraic and geometric topology (New Brunswick, N.J., 1983), Lecture Notes in Math. 1126, Springer-Verlag , Berlin 1985, 318-419. Zbl 0579.18006 MR 802796

[33] C. Weibel, Introduction to homological algebra. Cambridge Stud. Adv. Math. 38, Cambridge University Press, Cambridge 1994. Zbl 0797.18001 MR 1269324

Received April 13, 2010

G. Tabuada, Department of Mathematics, Massachusetts Institute of Technology, 77 Massachusetts Avenue, Cambridge, MA 02139-4307, U.S.A., and Departamento de Matemática e CMA, FCT-UNL, Quinta da Torre, 2829-516 Caparica, Portugal

E-mail: tabuada@math.mit.edu; tabuada@fct.unl.pt 